Supporting Information

\title{
Identification of a Stable Ozonide Ion Bound to a Single Cadmium Site within the Zeolite Cavity
}

Akira Oda, ${ }^{\dagger, * *}$ Kyoichi Sawabe, ${ }^{\dagger, *}$ Takahiro Ohkubo, ${ }^{\S}$ Yasushige Kuroda ${ }^{\S}$

'Department of Materials Chemistry, Graduate School of Engineering, Nagoya University, Furo-cho, Chikusa-ku,

Nagoya 464-8603, Japan.

\#Elements Strategy Initiative for Catalysts and Batteries (ESICB), Kyoto University, Kyoto 615-8520, Japan.

${ }^{\S}$ Department of Chemistry, Graduate School of Natural Science and Technology, Okayama University, 3-1-1 Tsushima, Kita-ku, Okayama 700-8530, Japan.

Corresponding Author

*E-mail: akira@chembio.nagoya-u.ac.jp (A. O.) 
Table S1. Comparison of the selected structural parameters of the $\left[\mathrm{CdO}_{3}\right]-\mathrm{Al}_{1} \mathrm{Si}_{4} \mathrm{O}_{4} \mathrm{H}_{12}$ cluster optimized at different computational levels. The relevant geometries were shown in Figure S1.

\begin{tabular}{ccccc}
\hline $\begin{array}{c}\text { Distance }(\AA), \\
\text { bond angle }\left({ }^{\circ}\right),\end{array}$ & $\begin{array}{c}{ }^{a} \text { B3LYP/def2- } \\
\text { TZVP, 6-31G(d) }\end{array}$ & $\begin{array}{c}{ }^{b} \text { B3LYP/def2- } \\
\text { TZVPD, 6-31G(d) }\end{array}$ & B3LYP/def2-TZVP & B3LYP/def2-QZVPP \\
\hline $\mathrm{Cd}-1 \mathrm{O}$ & 2.228 & 2.227 & 2.225 & \\
$\mathrm{Cd}-2 \mathrm{O}$ & 2.697 & 2.696 & 2.693 & 2.683 \\
$\mathrm{Cd}-3 \mathrm{O}$ & 2.252 & 2.250 & 2.248 & 2.237 \\
$1 \mathrm{O}-2 \mathrm{O}$ & 1.343 & 1.343 & 1.343 & 1.342 \\
$2 \mathrm{O}-3 \mathrm{O}$ & 1.336 & 1.336 & 1.336 & 1.336 \\
$1 \mathrm{O}-2 \mathrm{O}-3 \mathrm{O}$ & 111.8 & 111.8 & 111.8 & 111.7 \\
$1 \mathrm{O}-\mathrm{Cd}-3 \mathrm{O}$ & 59.4 & 59.4 & 59.5 & 59.7 \\
$\mathrm{Cd}-1 \mathrm{O}_{\mathrm{L}}$ & 2.204 & 2.203 & 2.213 & 2.206 \\
$\mathrm{Cd}-2 \mathrm{O}_{\mathrm{L}}$ & 2.206 & 2.208 & 2.216 & 2.208 \\
$1 \mathrm{O}_{\mathrm{L}}-\mathrm{Cd}-2 \mathrm{O}_{\mathrm{L}}$ & 71.8 & 71.8 & 71.5 & 71.7
\end{tabular}

${ }^{a}$ def2-TZVP basis set was used for $\mathrm{Cd}_{\text {, and }} \mathrm{O}_{3}$ adduct. 6-31G(d) basis was used for other atoms.

${ }^{b}$ def2-TZVPD basis set ${ }^{1-3}$ was used for $\mathrm{Cd}$, and $\mathrm{O}_{3}$ adduct. 6-31G(d) basis was used for other atoms.

Table S2. The selected structural parameters of the periodic DFT models representing the $\mathrm{CdO}_{3}$ species stabilized at the framework $\mathrm{AlO}_{4}$ tetrahedra located at different crystallographic positions.

\begin{tabular}{|c|c|c|c|c|c|c|c|c|c|c|}
\hline \multirow{2}{*}{ Site } & \multicolumn{10}{|c|}{ Distance $(\AA)$, bond angle $\left(^{\circ}\right)$} \\
\hline & $\mathrm{Cd}-1 \mathrm{O}$ & $\mathrm{Cd}-2 \mathrm{O}$ & $\mathrm{Cd}-3 \mathrm{O}$ & $10-20$ & $2 \mathrm{O}-3 \mathrm{O}$ & $10-2 \mathrm{O}-3 \mathrm{O}$ & $1 \mathrm{O}-\mathrm{Cd}-3 \mathrm{O}$ & $\mathrm{Cd}-1 \mathrm{O}_{\mathrm{L}}$ & $\mathrm{Cd}-2 \mathrm{O}_{\mathrm{L}}$ & $1 \mathrm{O}_{\mathrm{L}}-\mathrm{Cd}-2 \mathrm{O}_{\mathrm{L}}$ \\
\hline $\mathrm{T} 1$ & 2.229 & 2.691 & 2.241 & 1.368 & 1.362 & 112.0 & 60.8 & 2.232 & 2.232 & 70.9 \\
\hline $\mathrm{T} 2$ & 2.225 & 2.701 & 2.260 & 1.370 & 1.360 & 111.9 & 60.6 & 2.233 & 2.231 & 70.9 \\
\hline $\mathrm{T} 3$ & 2.247 & 2.699 & 2.235 & 1.360 & 1.371 & 111.9 & 60.6 & 2.283 & 2.212 & 69.9 \\
\hline $\mathrm{T} 4$ & 2.198 & 2.694 & 2.280 & 1.376 & 1.355 & 112.1 & 60.8 & 2.243 & 2.262 & 71.4 \\
\hline T5 & 2.252 & 2.699 & 2.229 & 1.359 & 1.371 & 111.9 & 60.6 & 2.233 & 2.237 & 70.3 \\
\hline T6 & 2.254 & 2.699 & 2.228 & 1.361 & 1.370 & 111.9 & 60.6 & 2.265 & 2.246 & 70.7 \\
\hline $\mathrm{T} 7$ & 2.284 & 2.710 & 2.216 & 1.355 & 1.377 & 111.9 & 60.4 & 2.311 & 2.228 & 69.8 \\
\hline $\mathrm{T} 8$ & 2.309 & 2.723 & 2.212 & 1.351 & 1.380 & 111.8 & 60.0 & 2.311 & 2.268 & 68.7 \\
\hline T9 & 2.241 & 2.699 & 2.243 & 1.365 & 1.365 & 111.9 & 60.6 & 2.283 & 2.239 & 70.2 \\
\hline $\mathrm{T} 10$ & 2.219 & 2.697 & 2.260 & 1.373 & 1.358 & 111.9 & 60.7 & 2.252 & 2.228 & 71.4 \\
\hline T11 & 2.247 & 2.696 & 2.230 & 1.361 & 1.369 & 111.9 & 60.7 & 2.229 & 2.262 & 71.0 \\
\hline $\mathrm{T} 12$ & 2.268 & 2.694 & 2.209 & 1.356 & 1.375 & 112.1 & 60.8 & 2.250 & 2.223 & 70.5 \\
\hline Average & 2.248 & 2.700 & 2.237 & 1.362 & 1.368 & 111.9 & 60.6 & 2.260 & 2.239 & 70.5 \\
\hline $\begin{array}{l}\text { Standard } \\
\text { deviation }\end{array}$ & 0.029 & 0.008 & 0.020 & 0.007 & 0.007 & 0.1 & 0.2 & 0.029 & 0.017 & 0.7 \\
\hline
\end{tabular}


Table S3. The energy of the periodic DFT models representing the $\mathrm{CdO}_{3}$ species stabilized at the framework $\mathrm{AlO}_{4}$ tetrahedra located at different crystallographic positions.

\begin{tabular}{ccc}
\hline Site & Energy $(\mathrm{eV})$ & Relative energy $(\mathrm{eV})$ \\
\hline T1 & -2321.0112 & -0.0294 \\
T2 & -2320.9114 & 0.0704 \\
T3 & -2320.9175 & 0.0643 \\
T4 & -2321.0508 & -0.0690 \\
T5 & -2320.9818 & 0.0000 \\
T6 & -2320.7763 & 0.2055 \\
T7 & -2321.0306 & -0.0488 \\
T8 & -2321.0481 & -0.0663 \\
T9 & -2320.9966 & -0.0148 \\
T10 & -2320.9382 & 0.0436 \\
T11 & -2320.9167 & 0.0651 \\
T12 & -2321.0164 & -0.0346
\end{tabular}

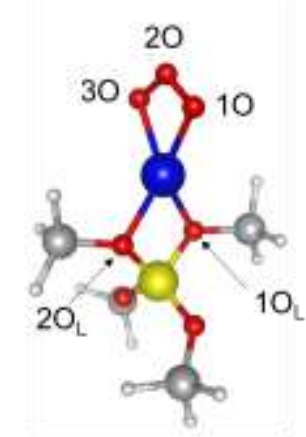

B3LYP/def-TZVP, 6-31G(d)

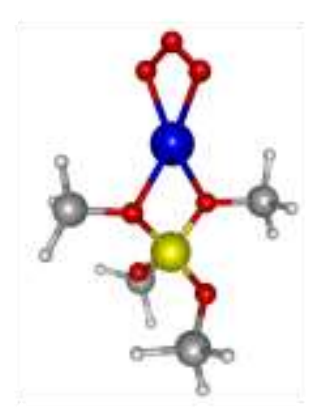

B3LYP/def-TZVPD, 6-31G(d)

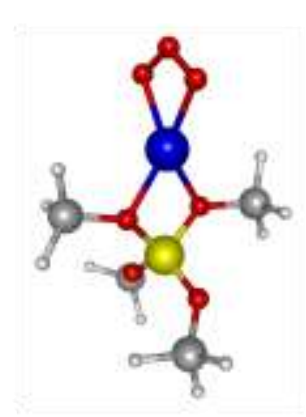

B3LYP/def-TZVP

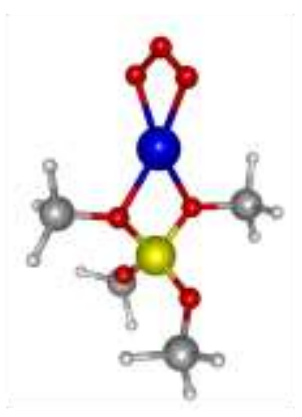

B3LYP/def-QZVPP

Figure S1. Comparison of the local geometries of the $\left[\mathrm{CdO}_{3}\right]-\mathrm{Al}_{1} \mathrm{Si}_{4} \mathrm{O}_{4} \mathrm{H}_{12}$ cluster optimized at different computational levels. The detailed structural parameters were summarized in Table S1.

\section{Supporting References}

1. Feller, D. The Role of Databases in Support of Computational Chemistry Calculations. J. Comput. Chem. 1996, $17,1571-1586$.

2. Schuchardt, K. L.; Didier, B. T.; Elsethagen, T.; Sun, L.; Gurumoorthi, V.; Chase, J.; Li, J.; Windus, T. L. Basis Set Exchange: A Community Database for Computational Sciences. J. Chem. Inf. Model. 2007, 47, 1045-1052.

3. Pritchard, B. P.; Altarawy, D.; Didier, B.; Gibson, T. D.; Windus, T. L. A New Basis Set Exchange: An Open, Upto-date Resource for the Molecular Sciences Community. J. Chem. Inf. Model. 2019, 59, 4814-4820. 